\title{
Wave power flux and ray-tracing in regions of resonant absorption
}

\author{
M D Tokman $\dagger$, E Westerhof $\ddagger$ and M A Gavrilova $\dagger$ \\ $\dagger$ Institute of Applied Physics of the Russian Academy of Sciences, 603600, Nizhny Novgorod, \\ Russia \\ \$ FOM-Instituut voor Plasmafysica 'Rijnhuizen', Associatie Euratom-FOM, \\ Trilateral Euregio Cluster, PO Box 1207, 3430 BE Nieuwegein, The Netherlands
}

Received 30 July 1999

\begin{abstract}
The propagation of waves in weakly dissipative plasmas is investigated. A new expression for the wave energy flux is obtained, which is proportional to $\partial \lambda^{\text {mode }} / \partial k$, where $\lambda^{\prime \text { mode }}$ is the real part of the eigenvalue of the dispersion tensor corresponding to the wave mode. Significant differences from the usual definition of dielectric wave energy flux occur in case of a non-negligible anti-Hermitian contribution to the dielectric tensor. This occurs, for example, near electron cyclotron resonance. The direction of the corrected wave energy flux is consistent with that of the trajectory of a wave beam crossing the EC resonance as obtained in Westerhof (Westerhof E 1997 Plasma Phys. Control. Fusion 39 1015). It is shown that ray-tracing near cyclotron resonance can be performed with the use of $\lambda^{\prime \text { mode }}$ as ray-Hamiltonian.
\end{abstract}

\section{Introduction}

Propagation of electromagnetic waves in media with resonant dissipation is a long-standing problem [1]. Recently, this problem has received renewed attention in connection with the problem of electromagnetic wave propagation through electron cyclotron resonant layers in a thermonuclear plasma (e.g. [2-11]). In most cases of practical interest the spatial dissipation remains small in the zone of cyclotron absorption, and one would expect most results and techniques of wave theory for weakly dissipative media to apply in the cyclotron resonance zone as well. In particular, provided the usual conditions for the applicability of the geometric optics or WKB approximation are satisfied, one would expect the expression for the dielectric wave energy flux to hold, and wave trajectories to be describable by ray-tracing techniques based on the real part of the dispersion relation alone $[12,13]$.

However, it appears that the situation in the cyclotron resonance zone is not so simple: the anti-Hermitian parts of the dielectric tensor elements $\varepsilon_{n m}$ are of the same order of magnitude there as the Hermitian parts and both may be very much larger than unity. That the spatial dissipation is weak nevertheless is due to the specific polarization of the normal waves [5, 14]. The anti-Hermitian part of the dielectric tensor influences in a fundamental way both the wave dispersion and wave polarization. Still, as shown in $[10,11,15]$, wave propagation can be described properly by means of ray-tracing with the natural proviso that the real part of the wavevector that is obtained should coincide (at least approximately) with the real part of the wave vector as obtained from the full, complex dispersion relation. In $[11,15]$ this is achieved by construction rather than by approximation of the dispersion relation. As shown by Piliya and Fedorov [5], the non-negligible anti-Hermitian part of the dielectric tensor leads to an 
additional term in the Poynting theorem averaged over a wave period, i.e. in the power balance equation. This invalidates the usual expression for the wave energy flux in the cyclotron resonance zone. The latter is indeed found to provide an improper description of the direction of wave propagation [11].

In the present paper, it is shown that the additional term introduced in the power balance equation by Piliya and Fedorov can by rewritten in such a way that the power balance again takes the form of the divergence of an energy flux balanced by a single source term (section 2). This then leads to a new expression for the wave energy flux, which is found to be proportional to the wave vector derivative of the eigenvalue $\lambda^{\text {mode }}$ that corresponds to the wave mode under consideration, of the dispersion tensor

$$
D_{n m}=\delta_{n m} k^{2}-k_{n} k_{m}-\frac{\omega^{2}}{c^{2}} \varepsilon_{n m}
$$

where $\delta_{n m}$ is the unit tensor, $\boldsymbol{k}$ is the wave vector, $\omega$ is the wave frequency, $c$ is the speed of light, and $\varepsilon_{n m}$ is the dielectric tensor. This new expression is shown to be consistent with the results on wave propagation of [11]. It also suggests the use of the eigenvalue as the Hamiltonian for ray-tracing, an option that is explored in section 3. A brief summary and the conclusions are given in section 4 .

\section{Wave power flux}

The starting point of the discussion of the wave power flux is, as usual, the Poynting theorem in the form as it follows directly from Maxwell's equations:

$$
\frac{1}{8 \pi} \frac{\partial}{\partial t}\left(\tilde{\boldsymbol{B}}^{2}+\tilde{\boldsymbol{E}}^{2}\right)+\frac{1}{4 \pi} \frac{\partial}{\partial \boldsymbol{r}} \cdot(\tilde{\boldsymbol{E}} \times \tilde{\boldsymbol{B}})+\tilde{\boldsymbol{j}} \cdot \tilde{\boldsymbol{E}}=0
$$

where $\tilde{E}$ and $\tilde{B}$ are the fluctuating electric and magnetic fields of the wave, and $\tilde{j}$ is the fluctuating current density of the plasma in response to the wave fields. Because we are interested in the trajectory of quasi-stationary wave beams and the wave power flux rather than the wave power density, we restrict the following discussion to the time independent case, i.e., neglecting the first term in the Poynting theorem (2).

We consider the propagation through a homogeneous, weakly dissipative medium of a quasi-monochromatic, stationary wave of the following form

$$
\tilde{\boldsymbol{E}}=\boldsymbol{E}(\boldsymbol{r}) \exp \left(\mathrm{i} \boldsymbol{k}_{0} \cdot \boldsymbol{r}-\mathrm{i} \omega t\right)
$$

where $\boldsymbol{E}(\boldsymbol{r})$ is the weakly varying amplitude of the wave field, $\boldsymbol{k}_{0}$ is the real wave vector, and $\omega$ is the wave frequency. Wave absorption is described by the spatial dependence $E(r)$. Note, that the requirement for the applicability of geometric optics or WKB, $k_{0} \gg|(\partial E / \partial r) / E|$, implies weak absorption. Next, the procedure given by Piliya and Fedorov [5] for the case of non-vanishing anti-Hermitian components of the dielectric tensor is followed: by averaging the Poynting theorem over a wave period they obtain the power balance equation in the form

$$
\nabla \cdot S+Q+q=0
$$

where (note the convention of summation over repeated indices)

$\boldsymbol{S}=\frac{c}{8 \pi} \operatorname{Re}\left(\boldsymbol{E} \times \boldsymbol{B}^{*}\right)-\left.\frac{\omega}{16 \pi}\left(\frac{\partial \varepsilon_{n m}^{H}}{\partial \boldsymbol{k}}\right)\right|_{k=k_{0}} E_{n}^{*} E_{m}=\left.\frac{c^{2}}{16 \pi \omega} \frac{\partial}{\partial \boldsymbol{k}}\left(D_{n m}^{H}\right)\right|_{k=k_{0}} E_{n}^{*} E_{m}$

is the standard expression for the dielectric energy flux $[12,13]$ in a dispersive medium, and

$$
Q=-\left.\frac{\mathrm{i}}{8 \pi}\left(\varepsilon_{n m}^{a H}\right)\right|_{k=k_{0}} E_{n}^{*} E_{m}=\left.\frac{\mathrm{i} c^{2}}{8 \pi \omega}\left(D_{n m}^{a H}\right)\right|_{k=k_{0}} E_{n}^{*} E_{m}
$$


is the standard expression for the energy losses of the wave to the medium $[12,13]$, while the last term

$$
\begin{aligned}
q & =-\left.\frac{\omega}{16 \pi}\left(\frac{\partial \varepsilon_{n m}^{a H}}{\partial \boldsymbol{k}}\right)\right|_{k=k_{0}} \cdot\left(E_{n}^{*} \frac{\partial E_{m}}{\partial \boldsymbol{r}}-\frac{\partial E_{n}^{*}}{\partial \boldsymbol{r}} E_{m}\right) \\
& =\left.\frac{c^{2}}{16 \pi \omega}\left(\frac{\partial D_{n m}^{a H}}{\partial \boldsymbol{k}}\right)\right|_{k=k_{0}} \cdot\left(E_{n}^{*} \frac{\partial E_{m}}{\partial \boldsymbol{r}}-\frac{\partial E_{n}^{*}}{\partial \boldsymbol{r}} E_{m}\right)
\end{aligned}
$$

arises in dissipative media with a non-negligible anti-Hermitian part of the dielectric tensor. The presence of this last term $q$ shows that it is not allowed to identify the vector $S$ with the wave energy flux when the anti-Hermitian elements of the dielectric tensor $\varepsilon_{n m}^{a H}$ do not vanish.

However, with the help of the method as outlined in [10], it is possible to rewrite the expression (4) in the standard form of the wave power balance: that is in the form of the divergence of a flux and a single source term. In order to show this, the wave field (3) is written as a composition of normal modes by

$$
\boldsymbol{E}(\boldsymbol{r})=\int \boldsymbol{e}\left(\lambda^{\text {mode }}\right) A_{k}\left(\boldsymbol{k}_{0}+\Delta \boldsymbol{k}\right) \delta\left(\lambda^{\text {mode }}\right) \exp (\mathrm{i} \Delta \boldsymbol{k} \cdot \boldsymbol{r}) \mathrm{d} \Delta \boldsymbol{k}
$$

Here, $\lambda^{\text {mode }}$ is the eigenvalue of the dispersion tensor $D_{n m}\left(\boldsymbol{k}_{0}+\Delta \boldsymbol{k}\right)$ corresponding to the wave mode under consideration, which has a dispersion relation $\lambda^{\text {mode }}=0$, and $e\left(\lambda^{\text {mode }}\right)$ is the associated unit eigenvector. The spectral density of normal modes is equal to $A_{k}\left(\boldsymbol{k}_{0}+\Delta \boldsymbol{k}\right) \delta\left(\lambda^{\text {mode }}\right)$, where the Dirac delta function excludes contributions from $\Delta \boldsymbol{k}$ values that do not satisfy the dispersion relation, and $A_{k}$ is the appropriate weight function. Taking into account the narrowness of the spatial spectrum, $k_{0} \gg \Delta k$, the slowly varying part of the wave field, $\boldsymbol{E}(\boldsymbol{r})$, may be written as

$$
\boldsymbol{E}(\boldsymbol{r})=e\left(\boldsymbol{k}_{0}\right) A(\boldsymbol{r})-\left.\mathrm{i} \frac{\partial A}{\partial r_{n}}\left(\frac{\partial \boldsymbol{e}}{\partial k_{n}}\right)\right|_{\boldsymbol{k}=k_{0}}
$$

where $\boldsymbol{e}\left(\boldsymbol{k}_{0}\right)$ is the unit eigenvector (or polarization vector) for the eigenvalue $\lambda^{\text {mode }}\left(\boldsymbol{k}_{0}\right)$, and $A(r)$ is the slowly varying scalar amplitude of the wave field defined by

$$
A(r)=\int A_{k}\left(\boldsymbol{k}_{0}+\Delta \boldsymbol{k}\right) \delta\left(\lambda^{\text {mode }}\right) \exp (\mathrm{i} \Delta \boldsymbol{k} \cdot \boldsymbol{r}) \mathrm{d} \Delta \boldsymbol{k} .
$$

Note that the second term on the right hand side of equation (9) describes, in particular, a possible influence of wave absorption on the wave polarization.

Next, the expression (9) is substituted into equations (4)-(7), and the slowly varying scalar amplitude is represented as $A=|A| \operatorname{expi} \phi$ with slowly varying relative phase $\phi$. Since the spatial dependence of $A(r)$ is weak, terms proportional to second order spatial derivatives, $\partial^{2} A / \partial r^{2}$, or squared first-order derivatives, $(\partial A / \partial r)^{2}$, are neglected with the result

$$
\begin{aligned}
\frac{16 \pi \omega}{c^{2}}(\nabla \cdot S+ & Q+q)=\frac{\partial}{\partial r_{j}}\left(|A|^{2} \frac{\partial D_{n m}^{H}}{\partial k_{j}} e_{n}^{*} e_{m}\right)+2 \mathrm{i}|A|^{2} D_{n m}^{a H} e_{n}^{*} e_{m} \\
& +2 \mathrm{i}|A|^{2} \frac{\partial \phi}{\partial r_{j}} D_{n m}^{a H}\left(e_{n}^{*} \frac{\partial e_{m}}{\partial k_{j}}+\frac{\partial e_{n}^{*}}{\partial k_{j}} e_{m}\right)+\frac{\partial|A|^{2}}{\partial r_{j}} D_{n m}^{a H}\left(e_{n}^{*} \frac{\partial e_{m}}{\partial k_{j}}-\frac{\partial e_{n}^{*}}{\partial k_{j}} e_{m}\right) \\
& +2 \mathrm{i}|A|^{2} \frac{\partial \phi}{\partial r_{j}} \frac{\partial D_{n m}^{a H}}{\partial k_{j}} e_{n}^{*} e_{m}
\end{aligned}
$$

where the first term on the right-hand side comes from $S$, the second to fourth terms originate from $Q$, and the final term is the only contribution that remains from $q$. Taking into account the relations for the polarization vector

$$
D_{n m}^{a H} e_{m}=-D_{n m}^{H} e_{m} \quad e_{n}^{*} D_{n m}^{a H}=e_{n}^{*} D_{n m}^{H}
$$


to rewrite the fourth term on the right-hand side, this term combines with the first into the divergence of a flux, while the remaining terms can be combined into a source term [10]. As a result, the power balance is again cast in the standard form

$$
\nabla \cdot \tilde{\boldsymbol{S}}+\tilde{Q}=0
$$

with the corrected dielectric wave energy flux

$$
\tilde{\boldsymbol{S}}=\left.\frac{c^{2}}{16 \pi \omega}|A|^{2} \frac{\partial}{\partial \boldsymbol{k}}\left(D_{n m}^{H} e_{n}^{*} e_{m}\right)\right|_{k=k_{0}}
$$

and the corrected source term [10]

$$
\begin{aligned}
\tilde{Q} & =\frac{\mathrm{i} c^{2}}{8 \pi \omega}|A|^{2}\left[\left(1+\frac{\partial \phi}{\partial r_{j}} \frac{\partial}{\partial k_{j}}\right) e_{n}^{*} e_{m} D_{n m}^{a H}\right]_{k=k_{0}} \\
& \approx \frac{\mathrm{i} c^{2}}{8 \pi \omega}|A|^{2}\left(e_{n}^{*} e_{m} D_{n m}^{a H}\right)_{k=k_{0}+\nabla \phi} .
\end{aligned}
$$

The equation for the corrected wave energy flux is the main result of the present paper.

Note that equation (13) differs with respect to the standard expression for the dielectric energy flux [12] in the application of the wave vector derivative not only to the dispersion tensor but also to the unit eigenvector and its complex conjugate. Although the anti-Hermitian part of the dispersion tensor does not appear explicitly, it does affect the wave flux (13) by its effect on the wave polarization, which follows immediately from the relations (11). Near cyclotron resonances the wave polarization depends sensitively on the wave vector and, consequently, in those regions the wave flux (13) is expected to differ significantly from its standard expression. When the effect of the anti-Hermitian part on the wave polarization would be negligible and, consequently, the relation $D_{m n}^{H} e_{n}=0$ would hold at least approximately (instead of (11)), the wave energy flux (13) reduces to the standard expression (5).

The present result is in agreement with the results obtained previously by Westerhof [11] on wave propagation through electron cyclotron resonant layers. This is illustrated in figure 1, which shows the direction of propagation of the maximum of a wave beam through cyclotron resonance as obtained in [11], compared with both the standard expression for the dielectric energy flux and the corrected expression for the wave energy flux (13). Examples for both $\mathrm{O}$-mode and X-mode propagation through the fundamental electron cyclotron resonance are shown. The direction of the standard wave energy flux (5) strongly deviates from the direction of propagation of the wave beam. In contrast, the direction of the corrected wave flux (13) is very close to or practically overlaps with the results from [11]. The small remaining difference in figure $1(a)$ is due to the fact that the assumption of weak absorption $\operatorname{Im} k \ll \operatorname{Re} k$ is only marginally satisfied in this case of an O-mode wave propagating nearly perpendicularly to the equilibrium magnetic field.

\section{Ray-tracing}

Note that the term between the brackets in the modified expression of the wave energy flux is in fact identical to the real part of the eigenvalue of the particular mode under consideration:

$$
\lambda^{\prime \text { mode }}=D_{n m}^{H} e_{n}^{*} e_{m} .
$$

Here, a prime (double prime) is used to indicate the real (imaginary) part. Consequently, the real part of the eigenvalue can serve as the Hamiltonian for ray-tracing:

$$
\frac{\mathrm{d} \boldsymbol{r}}{\mathrm{d} \tau}=\frac{\partial \lambda^{\text {mode }}}{\partial \boldsymbol{k}} \quad \frac{\mathrm{d} \boldsymbol{k}}{\mathrm{d} \tau}=-\frac{\partial \lambda^{\prime \text { mode }}}{\partial \boldsymbol{r}}
$$



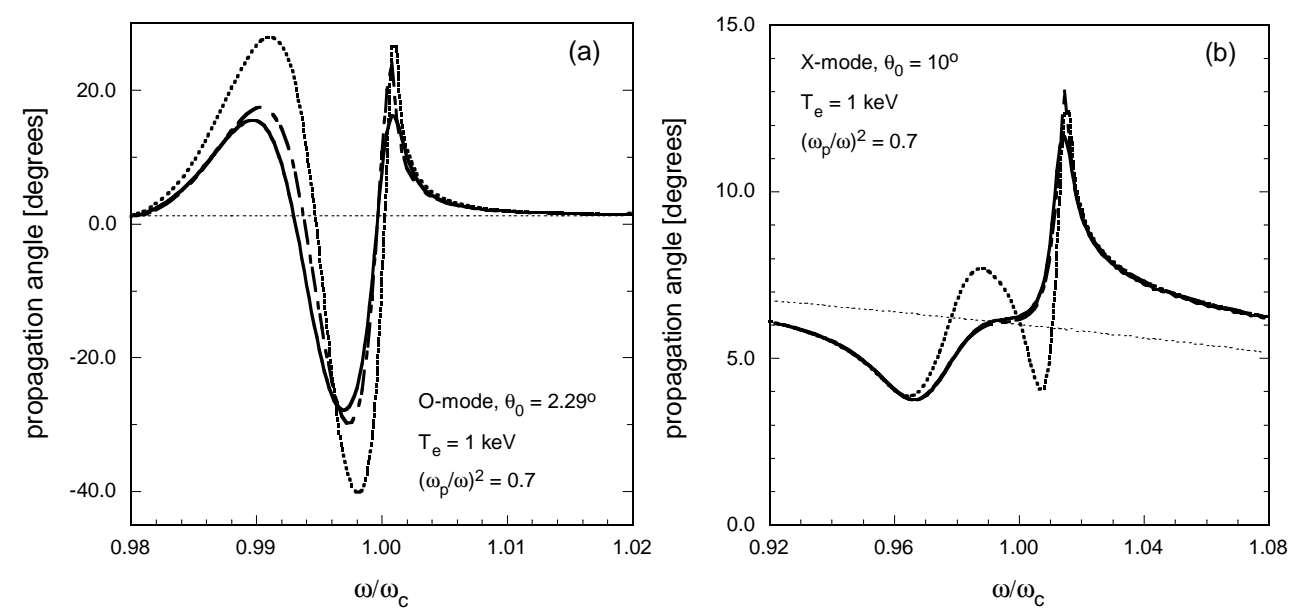

Figure 1. The angle of wave propagation with respect to the normal of the magnetic field: $(a)$ for O-mode injected at an angle of $2.29^{\circ} ;(b)$ for X-mode injected at $10^{\circ}$. Full curves show the results of [11], dotted curves show the direction of the standard wave flux in equation (5), and dashed curves show the direction of the modified wave flux in equation (13). Thin dotted lines represent results from the cold plasma approximation.

where the initial conditions must correspond to the solution of the dispersion equation $\lambda^{\text {mode }}\left(\boldsymbol{r}\left(\tau_{0}\right), \boldsymbol{k}\left(\tau_{0}\right)\right)=0$.

In figure 2 the ray-trajectories are shown as obtained from the ray-tracing equations (16) for the same parameters as those used in figure 1. For comparison, the trajectories of the maximum of a corresponding wave beam are shown as obtained in [11]. A discrepancy is again found in figure 2(a) for the O-mode case due to the relatively strong absorption, which this time is larger as the difference in the direction of wave propagation is now integrated along the ray-trajectory. Also, the value of $\lambda^{\prime \text { mode }}$ is not conserved exactly along the ray-trajectory in the region of wave absorption. Almost perfect agreement with the results of [11] would be obtained if one corrected the eigenvalue for the presence of a finite imaginary part of the wave vector: that is to use for ray-tracing $\lambda^{\prime \text { mode }}\left(\boldsymbol{x}, \boldsymbol{k}^{\prime} ; k_{\perp}^{\prime \prime}\right)$ with $k_{\perp}^{\prime \prime}$ determined by some auxiliary relation. For example, the imaginary part of the wave vector could be obtained from the relation

$$
k_{\perp}^{\prime \prime} \frac{\partial \lambda^{\text {mode }}\left(\boldsymbol{r}, \boldsymbol{k}^{\prime}\right)}{\partial k_{\perp}}=\lambda^{\prime \text { mode }}\left(\boldsymbol{r}, \boldsymbol{k}^{\prime}\right)
$$

which is in fact identical to the power balance equation (4). Also, the value of $\lambda^{\text {mode }}$ would again be properly conserved. Still, such a further correction of the wave energy flux means the inclusion of higher order terms in the geometric optics approximation, and awaits a more formal derivation.

In principle one could also substitute in this paragraph the determinant at the dispersion tensor for the eigenvalue of the wave mode, i.e. the product of all three eigenvalues $D=\Pi_{i=1,3} \lambda_{i}$. However, in case of finite dissipation $\lambda^{\prime \text { mode }}\left(\boldsymbol{x}, \boldsymbol{k}^{\prime}\right)$ will become slightly nonzero as the ray is traced. Since in the case of $D$ it is multiplied by two potentially very large numbers, a small deviation in $\lambda^{\prime \text { mode }}$ can have large consequences for $D^{\prime}$ and may lead to unacceptable errors. Still, as with $\lambda^{\prime \text { mode }}$, a correction for a finite imaginary part of $k$ can be applied and the relation $D^{\prime}\left(\boldsymbol{x}, \boldsymbol{k}^{\prime} ; \boldsymbol{k}_{\perp}^{\prime \prime}\right)$ can be used as ray-Hamiltonian. The method for ray-tracing as proposed in [15] can be regarded as an example of the latter. 

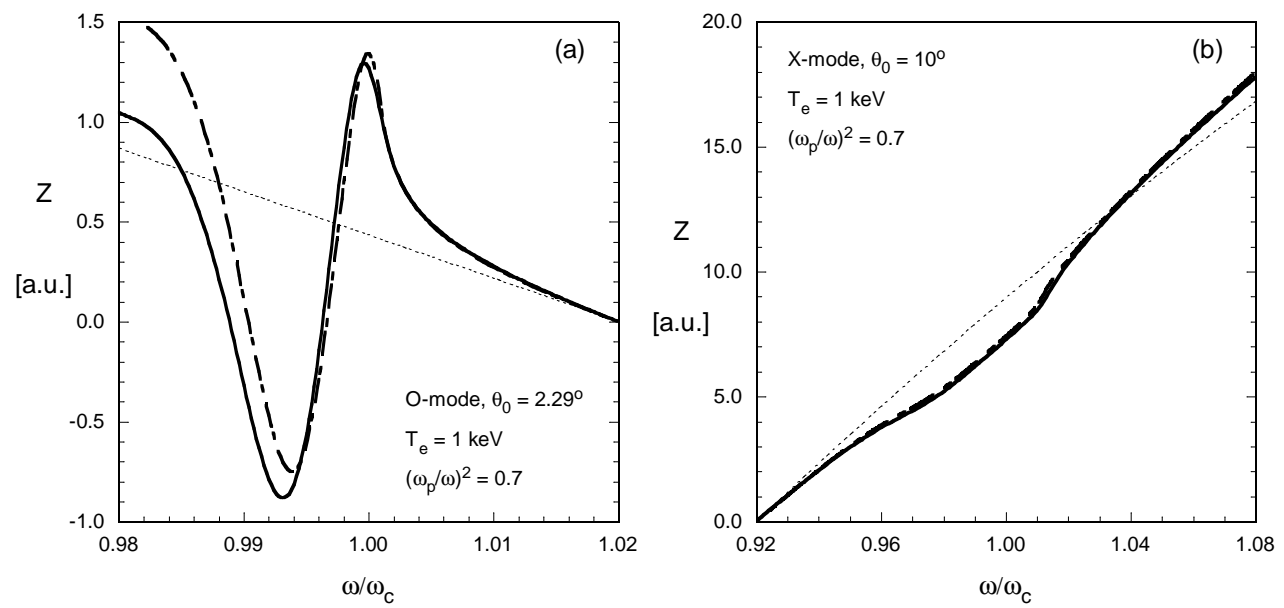

Figure 2. The trajectory of a wave beam: (a) for O-mode injected from the low field side at an angle of $2.29^{\circ} ;(b)$ for X-mode injected from the high field side with $10^{\circ}$. Full curves show the results of [11], and dashed curves the results of ray-tracing using equations (16). Thin dotted lines represent results from the cold plasma approximation.

\section{Summary and discussion}

In the previous sections some peculiarities of wave propagation in media with resonance dissipation have been discussed. It is shown that in spite of large anti-Hermitian components of the dielectric tensor and large wave vector derivatives of both the Hermitian and antiHermitian parts of the dielectric tensor, a proper wave power balance can be formulated. This leads, however, to the necessity of a redetermination of the wave energy flux, even in cases of relatively weak absorption. Here, weak absorption is not synonymous with a small anti-Hermitian part of the dielectric tensor. Quite the opposite is often true near cyclotron resonances. The direction of the correct wave energy flux (13) agrees well with the direction of propagation of a wave beam as obtained previously in [11].

The results presented above also show that the effects of spatial dispersion can be accounted for in the usual frame work of geometrical optics, even in the regions of cyclotron resonant absorption. A proper ray-Hamiltonian for use in geometrical optics ray-tracing is the real part of that eigenvalue of the dispersion tensor corresponding to the wave mode under consideration, i.e. $\lambda^{\text {mode }}$. An alternate ray-Hamiltonian has been proposed in $[11,15]$, which is equally valid as it is constructed to reproduce the identical wave dispersion. The present results then provide a further confirmation of the effects of the wave dispersion near electron cyclotron resonance as reported earlier $[8,9,15]$.

The present results resolve a number of paradoxes in the applicability of the theory of wave propagation in weakly dissipative media to cyclotron resonance. Firstly, wave propagation can be described by ray-tracing based on the real part of the dispersion relation. After all, the dispersion relation for the mode can be written as $\lambda^{\text {mode }}=0$. Secondly, this is achieved by neglecting the anti-Hermitian part of the dielectric tensor. However, the anti-Hermitian part can only be neglected after the full dielectric tensor has been accounted for in bringing the dispersion tensor to diagonal form. Finally, the results are in agreement with the well known observation that the direction of wave propagation is perpendicular to the dispersion surface [13]. 
The problem of geometrical optics in plasmas with a significant anti-Hermitian part of the dielectric tensor has also been addressed by Friedland and Bernstein $[16,17]$. They also consider time variability and use a formulation with complex wave frequency. An expansion is then made near the real frequency making use of frequency derivatives of the dielectric tensor. In the case of cyclotron resonance such an expansion in frequency will easily break down as a consequence of the resonant contributions to the dielectric tensor. This problem is related to the difficulty of the definition of the wave energy density near cyclotron resonance and the interpretation of the group velocity [2,3]. In the present work these problems did not occur because only the time-independent case was considered. Clearly more work needs to be done in this respect.

Finally, it must be remarked that the discussions above assumed the validity of the WKB or geometrical optics approximation. In applying these results, for example, to present day tokamak devices this assumption could be violated as the cyclotron resonance zone is spatially very localized and wave properties might change on length scales comparable to the wavelength. In such cases the problem needs to be treated by full wave calculations as in [18], which tend to show that the expected effects from the spatial dispersion near the cyclotron resonance might be only partially realized.

\section{Acknowledgments}

The collaboration of which this paper is the result has been made possible by a grant from the International Centre for Advanced Studies (INCAS, grant No 98-2-01) in Nizhny Novgorod, Russia. EW wishes to express his sincere thanks for the hospitality of the staff and students of the Institute for Applied Physics and of the staff of INCAS during his visit to Nizhny Novgorod. The work of EW was performed under the Euratom-FOM Association agreement with financial support from NWO and Euratom. MDT has also been supported by INCAS (grant No 98-2-01). MAG is grateful for support to the Russian Foundation for Basic Research (project No 98-02-17204) and to the program of the Russian Ministry of Science 'Controlled thermonuclear fusion and plasma processes' for students and postgraduates (project No 370).

\section{References}

[1] Brillouin L 1960 Wave Propagation and Group Velocity (New York: Academic)

[2] Maroli C and Petrillo V 1981 Plasma Phys. 23671

[3] Tanaka M, Fujiwara M and Ikegami H 1981 J. Phys. Soc. Japan 81358

[4] Bornatici M and Engelmann F 1983 Comment. Plasma Phys. Control. Fusion 857

[5] Piliya A D and Fedorov V A 1987 Reviews of Plasma Physics vol 13, ed B B Kadomtsev (New York: Consultants Bureau) p 335

[6] Pesić A and Stojić S 1993 J. Phys. Soc. Japan 622359

[7] Pesić A 1996 Europhys. Lett. 35349

[8] Pochelon A et al 1993 20th EPS Conf. on Controlled Fusion and Plasma Physics (Lisboa) part III, p 1029

[9] Ségui J L, Michelot Y, Vézard D, Laurent L and Giruzzi G 1995 EC-9 Proc. 9th Joint Workshop on ECE and ECH (Borrego Springs, CA, 23-26 January, 1995) ed J Lohr (Singapore: World Scientific) p 223

[10] Smirnov A I and Tokman M D 1996 Sov. Phys.-JETP 83294

[11] Westerhof E 1997 Plasma Phys. Control. Fusion 391015

[12] Bekefi G 1966 Radiation Processes in Plasmas (New York: Wiley)

[13] Stix T H 1992 Waves in Plasmas (New York: American Institute of Physics) ch 4

[14] Alikaev V V, Litvak A G, Suvorov E V and Fraiman A A 1992 High Frequency Plasma Heating ed A G Litvak (New York: American Institute of Physics) pp 1-64

[15] Westerhof E 1997 Proc. 10th Joint Workshop on ECE and ECH (6-11 April, Ameland, The Netherlands, 1997) (Singapore: World Scientific) p 39 
[16] Friedland L and Bernstein I B 1980 Phys. Rev. A 221680

[17] Bernstein I B and Friedland L 1983 Handbook of Plasma Physics, vol 1 ed A A Galeev and R N Sudan (Amsterdam: North-Holland) ch 2.5

[18] McDonald D C, Cairns R A, Lashmore-Davies C N and Le Clair G 1998 Phys. Plasmas 5883 